\title{
Perceived symmetry and name matching
}

\author{
MAURICE HERSHENSON and JOAN RYDER \\ Brandeis University, Waltham, Massachusetts 02254
}

\begin{abstract}
Letter pairs made from the letters F, G, J, L, and $R$ in normal, reversed, or inverted orientations were used as stimuli in a name-matching experiment. When the same letter was used twice to form a pair, the different orientation combinations produced four types of symmetry: rotational, vertical axis bilateral, and translational symmetry, and a distorted version of horizontal axis bilateral symmetry. The pattern of results suggests that symmetry was used as a "diagnostic" when the presence or absence of symmetry was easily detected (same-letter stimuli with vertical axis bilateral and translational symmetry, and all differentletter stimuli) and that letter names were matched only for those pairs whose symmetry was not easily assessed (same-letter stimuli with horizontal axis bilateral and rotational symmetry).
\end{abstract}

Letter-pair configurations that produced perceived symmetry took longer to match than pairs that produced asymmetric configurations when subjects matched the pair elements on the basis of their "physical" characteristics (Herschenson \& Ryder, Note 1). The stimuli used to demonstrate this effect were letter pairs composed of the asymmetric letters $F, G, J, L$, and $R$ in normal, reversed, and inverted orientations. Different orientation combinations produced configurations that were symmetrical in different ways for stimuli composed of two of the same letters (see below for a detailed description of the stimuli).

When symmetry was perceived, both elements of the pair were seen as components of a single symmetrical structure. Since the task required comparison of the physical characteristics of the pair elements, the analysis was similar to that involved in separating and comparing "embedded" figures (Gottschaldt, 1926). ${ }^{1}$ Hershenson and Ryder (Note 1) concluded, therefore, that more time was needed to match symmetrical pairs because subjects had to break down the initial perceptual organization in order to obtain information about the physical properties of the individual pair members: the information necessary for a correct match. Thus, for the symmetrical stimuli, the analysis for the match began after the initial organization that produced the perception of a symmetrical configuration.

The experiment to be reported here assessed the effects of these perceived symmetries in a simple cognitive task. Name matching was selected because it requires contact with memory and because retrieval of the letter names should require separation of the letter elements from the perceptually organized symmetrical configuration in the same way as in the physical-match task. But

Requests for reprints should be sent to Maurice Hershenson, Department of Psychology, Brandeis University, Waltham, Massachusetts 02254 . it is not clear whether all symmetry types should affect a cognitive task in the same way. Hershenson and Ryder (Note 1) observed, for example, that it is sometimes necessary to instruct subjects to notice the fact that stimuli are symmetrical about a diagonal axis (e.g., Hock, 1973). Consequently, they suggested that, although physical symmetries of all types produce organized perceptual representations, they may not lead directly to knowledge of the fact that the figure is symmetrical. Thus, despite the fact that all types of symmetry directly affected the performance of a "perceptual" task (physical match), different types of symmetry may differentially affect processing in a cognitive task.

\section{METHOD}

\section{Stimuli}

The stimuli were letter pairs made from the asymmetrical uppercase letters $F, G, J, L$, and $R$ in normal $(N)$, reversed (R), or inverted (I) orientations. Four types of symmetry were produced by combining the same letters in all orientations in all possible ways: rotational (ROT), vertical axis bilateral (VAB), and translational (TRA) symmetry, and a representation of horizontal axis bilateral (HAB) symmetry that was distorted because the letters were side by side. When different letters in the corresponding orientations were combined, they produced a set of corresponding asymmetric stimuli. Table 1 contains examples of the letter-orientation combinations that produced the four types of symmetry and the corresponding asymmetric stimuli that were produced when different letters were used in the respective orientation combinations. Normal-normal stimulus pairs were included to provide sufficient stimuli to balance "same" and "different" responses, but they were not included in any of the analyses because pilot experiments showed that they produced an overriding affect of familiarity.

The four types of symmetry have been described objectively (Garner, 1974; Garner \& Clement, 1963; Vitz \& Todd, 1971; Weyl, 1952; Zusne, 1971). Weyl described three major types of symmetry, rotational symmetry, bilateral symmetry, and translational symmetry, that may be defined as follows: A spatial configuration is symmetric with respect to a point (i.e., possesses rotational symmetry) if there exists a point in the figure about which it may be rotated by less than $360 \mathrm{deg}$ to 
Table 1

Exemplars of the Letter-Orientation Combinations that Produced the Four Types of Symmetry in Same-Letter Pairs and Corresponding Asymmetric Stimuli Produced by Different-Letter Pairs

\begin{tabular}{cccc}
\hline $\begin{array}{c}\text { Symmetry } \\
\text { Type }\end{array}$ & $\begin{array}{c}\text { Letter- } \\
\text { Orientation } \\
\text { Combination }\end{array}$ & $\begin{array}{c}\text { Symmetric } \\
\text { (Same- } \\
\text { Letter) } \\
\text { Stimuli }\end{array}$ & $\begin{array}{c}\text { Corresponding } \\
\text { (Different- } \\
\text { Letter) } \\
\text { Stimuli }\end{array}$ \\
\hline ROT & RI & $7 t$ & 76 \\
& IR & $t 7$ & $t 9$ \\
VAB & NR & $F 7$ & $F A$ \\
& RN & $7 F$ & $7 R$ \\
HAB & NI & $F t$ & $F b$ \\
& IN & $t F$ & $t R$ \\
TRA & RR & 77 & 79 \\
& II & $t t$ & $t b$ \\
& NN & FF & $F R$ \\
\hline
\end{tabular}

Note-Symmetry types: $R O T=$ rotational, $V A B=$ vertical axis bilateral, $H A B=$ horizontal axis bilateral (distorted), $T R A=$ translational. Letter-orientation combinations: $R=$ reversed, $I=$ inverted,$\quad N=$ normal. Normal-normal stimuli were not included in the analyses.

reproduce the figure. A configuration is symmetric with respect to a line/plane (i.e., possesses bilateral symmetry) if it is carried into itself by reflection in the line/plane. Finally, a configuration possesses translational symmetry if a translation carries every point of one part of the configuration into corresponding points of another part of the configuration.

To determine if the objective symmetry structure of the same-letter pairs produced consistent behavioral (perceptual or judgmental) differences, Hershenson and Ryder (Note 1) had the stimuli rated by two groups, one instructed to rate the "degree of symmetry" of each configuration (i.e., whole stimulus pair), and the other instructed to rate the "degree of organization and structure" of each configuration. Both instructions produced large differences between the ratings for symmetrical and asymmetrical stimuli. The same-letter stimuli were also subjected to ratings under the two instructions without the different-letter stimuli. The different instructions produced differences in the way the symmetries were rated: VAB was rated highest and $\mathrm{HAB}$ was rated lowest under both instructions, and ROT was rated higher than TRA when the instruction included the term "symmetry," but the opposite was true when the instruction only referred to "organization and structure." These outcomes were not surprising because many authors have noted that it is VAB symmetry that is usually noticed or called "symmetry" (e.g., Mach, 1897; Rock, 1973). Moreover, $\mathrm{HAB}$ is a distorted representation of the true symmetry and TRA is a repetitive pattern that, while highly organized and symmetrical in the formal sense, is not considered symmetrical in the popular sense (see Hershenson \& Ryder, Note 1 , for details of the ratings).

The stimulus pairs were lettered in black on white paper using LeRoy pen No. 3 and stencils No. $0250-240 \mathrm{C}$ for normal letters and No. 0550-240C for reversed and inverted letters. No letter subtended more than about $.5 \mathrm{deg}$ of arc in any of its dimensions. The letters were separated by a space of approximately $.25 \mathrm{deg}$ of arc, making the entire display less than $1.5 \mathrm{deg}$ of horizontal visual angle. The luminances of the stimulus and adapting fields were approximately $65 \mathrm{~cd} / \mathrm{m}^{2}$. There were 120 stimuli: 60 same-letter pairs composed of 30 pairs with the same letters in the same orientations and 30 pairs with the same letters in different orientations, and 60 different-letter pairs composed of 30 pairs with different letters in the same orientations and 30 pairs with different letters in different orientations.

\section{Apparatus and Procedures}

The stimuli were exposed for $100 \mathrm{msec}$ in one channel of a three-field tachistoscope (Scientific Prototype Model GB). A second channel, containing two faint lines, each bisected by a dot, served as an adapting field and identified the area within which the stimuli would appear. Subjects were instructed to fixate between the dots. Each trial started with a .5 -sec warning tone. The stimulus pair was exposed $.5 \mathrm{sec}$ after the offset of the tone. The fixation field was on between trials. Voice reaction time was measured to the nearest $1 \mathrm{msec}$ from the onset of the stimulus field using a Grason-Stadler voice-operated relay (Model E7300A-1) and a Hunter counter (Model 1520).

The 60 practice trials were of the same form as the experimental trails, except that the stimuli were composed of the letters A, B, K, T, and Y. Subjects were instructed to match the letters on the basis of letter names regardless of orientation as quickly and accurately as possible. Thus, to be correct, subjects were to respond "same" to all same-letter pairs and "different" to all different-letter pairs, regardless of the orientation of the letter components. (This is different from the physical-match experiment, in which subjects were correct when they said "same" only when the letters were in the same orientation.)

\section{Subjects}

Four graduate students served as subjects. Each had experience in reaction time experiments and had normal or corrected vision.

\section{RESULTS AND DISCUSSION}

An analysis of variance was computed on reciprocals of mean correct response latency scores for each subject per cell for the two orientation combinations in each symmetry type by same vs. different letter cell. The error rate was less than $2 \%$ throughout. Figure 1 shows the mean correct response latency for each symmetry type for symmetric (same-letter) and corresponding

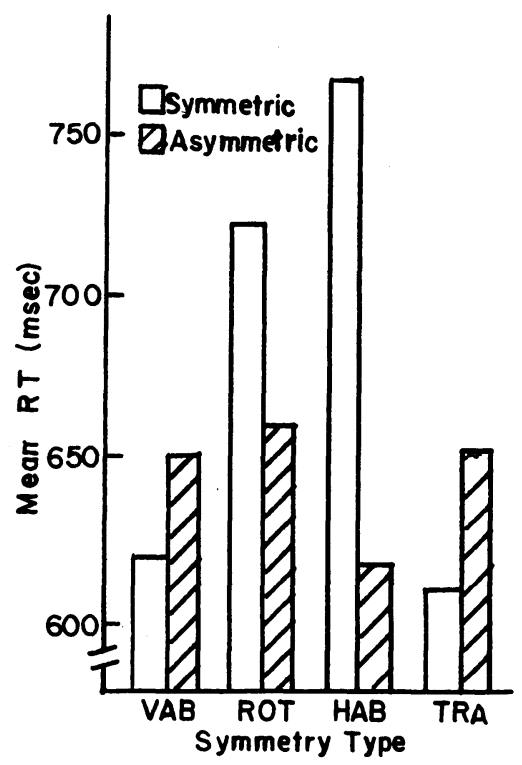

Figure 1. Mean correct response latency (in milliseconds) for the four types of symmetry for symmetric (same-letter) and corresponding asymmetric (different-letter) stimuli for the name-matching task. 
asymmetric (different-letter) stimuli $[F(3,9)=8.54$, $\mathrm{p}<.01]$. The fact that symmetry type was important $[F(3,9)=7.90, p<.01]$, whereas the presence or absence of symmetry was not $[F(1,3)=1.10, p>.05]$, can be seen in the figure. Long response latencies were produced only when same-letter HAB and ROT stimuli were processed; there were no significant differences among the response latencies produced by the other stimuli (Newman-Keuls test, $p>.05$ ). The stimuli showing $\mathrm{HAB}$ symmetry produced response latencies that were significantly longer than all others except those produced by same-letter ROT stimuli. The response latency for this (ROT) stimulus was significantly longer than only the three fastest mean response latencies (Newman-Keuls test, $\mathrm{p}<.05$ ).

The long latencies for the same-letter HAB and ROT pairs were probably not due to the presence of bilateral or rotational symmetry. It is difficult to find a reason why these symmetries should have affected naming any more or less than VAB or TRA symmetry. Indeed, if any of the symmetries would have been expected to produce strong inhibition due to perceptual organization, it would have been VAB (Corballis \& Beale, 1976; Corballis \& Roldan, 1974; Goldmeier, 1937/1972; Mach, 1897; Rock, 1973). In fact, among all the stimulus pairs, VAB was rated highest in all the subjective ratings of "symmetry" and "organization and structure" (Hershenson \& Ryder, Note 1).

It seems more likely that the differences were a function of the particular combinations of letter orientations that constituted these particular letter pairs. Analysis of the orientation combinations that produced these symmetries shows that HAB and ROT stimuli were the only same-letter pairs that contained one letter in the inverted orientation (normal inverted or inverted normal and reversed inverted or inverted reversed for $\mathrm{HAB}$ and ROT, respectively). But it was not simply the presence of an inverted letter that produced longer latencies; responses to pairs that contained two inverted letters were as fast as responses to any of the other pairs regardless of whether the letters were the same or were different. Moreover, if one of the letters was inverted but the two letters were different, the pair was also matched rapidly. Response latencies were long only for same-letter stimuli containing one inverted letter, or, to put it another way, the outcome that took longest to process was the determination of sameness when one of the letters of a same-letter pair was inverted.

Why, then, did these pairs take so long to match? One way to understand the overall pattern of results is suggested by the possibility that subjects did not always match on the basis of letter names but used symmetry as a "diagnostic" (Fox, 1975) for an initial quick response when the "symmetry" or "asymmetry" was easy to determine (same-letter VAB and TRA and all different-letter stimuli). When the analysis of symmetry was not easily determined (ROT and HAB), an additional stage of processing was invoked to make a more careful comparison on the basis of letter-name information.

This suggested sequence of processes is outlined in Figure 2. It shows an initial stage of processing in which the total configuration is assessed, that is, in which there is no need to break the configuration apart into its letter-element components to determine that sameletter VAB and TRA stimuli are symmetrical (in the broad sense) and that the different-letter stimuli are not. This initial determination can lead to fast "samedifferent" differentiation of all but the same-letter ROT and HAB stimuli, pairs whose symmetrical properties may not be so easily determined. Indeed, this possibility was suggested in the subjective ratings of these stimuli previously obtained; when instructed to rate on the basis of "organization and structure." subjects rated same-letter VAB and TRA higher than $\mathrm{HAB}$ and ROT when same-letter stimuli were presented alone as well as when they were presented along with the different-letter stimuli.

The figure also shows a second stage of processing for the same-letter ROT and HAB stimuli that probably involves letter-name retrieval and matching. It suggests that additional time is necessary for the operation of the second stage. Moreover, the amount of time necessary to obtain the letter names is different for the two array types because it takes longer to name letters that are rotated from a backward orientation than to name letters that are rotated from a normal orientation (Corballis \& Nagourney, 1978; Corballis, Zbrodoff,

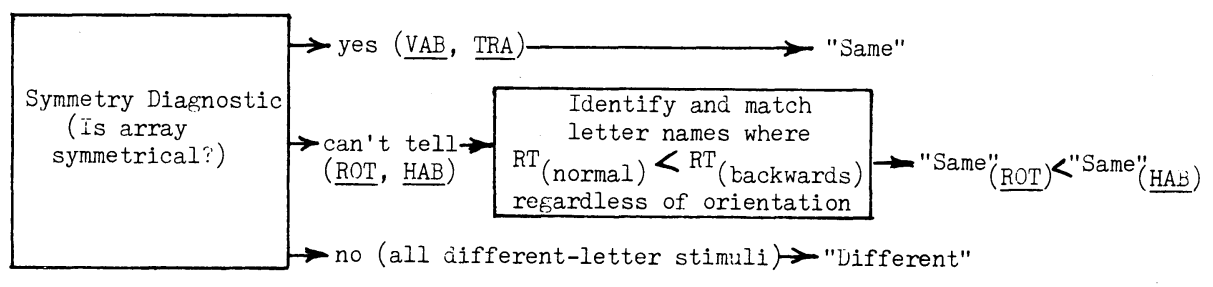

$$
\text { Stage } 1
$$

Figure 2. A possible sequence of processes that could have produced the data of Figure 1. In the first stage, symmetry is used as a diagnostic to produce fast "same" and "different" responses. In the second stage, letter names are obtained and matched. 
Shetzer, \& Butler, 1978; White, 1980). Thus, if the model is correct and letter names were retrieved and matched in the second stage of processing, one would expect it to take longer to say "same" to the same-letter HAB pair than to the same-letter ROT pair because the former contains a rotated backward letter whereas the latter contains a rotated normal letter.

Thus, it appears that perceptual organization was used as a diagnostic despite the fact that subjects were explicitly instructed to match on the basis of letter names. Consequently, this experiment did not produce the inhibition manifested when the same stimuli were used in a physical matching task. In a sense, then, the perceived symmetry may be said to have facilitated the matching process. It is more accurate, however, simply to describe the sequence of processing in terms of the two stages without describing first-stage processing as facilitation.

In conclusion, it should be noted that this two-stage model bears some resemblance to one previously proposed for visual matching when the stimuli could be separated into an easy-decision set, matched on the basis of a different criterion (Kroll \& Hershenson, 1980; Ryder, 1979; Rosen \& Hershenson, Note 2). That model also consisted of a first-stage processor that made decisions on the basis of holistic ("organizational") properties and a second-stage processor that made decisions on the basis of a more analytic treatment of the stimuli.

\section{REFERENCE NOTES}

1. Hershenson, M., \& Ryder, J. Perceived symmetry and visual matching. Manuscript submitted for publication, 1981.

2. Rosen, K., \& Hershenson, M. A test of the two-stage model of matching using "global" and "serial" configurations. Paper presented at the meeting of the Eastern Psychological Association, Hartford, Conn., April 1980.

\section{REFERENCES}

Corballis, M. C., \& Beale, I. L. The psychology of left and right. Hillsdale, N.J: Erlbaum, 1976.

Corballis, M. C., \& Nagourney, B. Latency to categorize disoriented alphanumeric characters as letters or digits. Canadian Journal of Psychology, 1978, 32, 186-188.

Corballis, M. C., \& Roldan, C. E. On the perception of symmetrical and repeated patterns. Perception \& Psychophysics, 1974, 16, 136-142.

Corballis, M. C., Zbrodoff, N. J., Shetzer, L. I., \& Butler, P. B. Decisions about identity and orientation of rotated letters and digits. Memory \& Cognition, 1978, 6, 98-107.

Egeth, H. E., Brownell, H. H., \& Geoffrion, L. D. Testing the role of vertical symmetry in letter matching. Journal of Experimental Psychology: Human Perception and Performance, 1976, 2, 429-434.

Fox, J. The use of structural diagnostics in recognition. Journal of Experimental Psychology: Human Perception and Performance, 1975, 1, 57-67.

GARNER, W. R. The processing of information and structure. New York: Erlbaum, 1974.

Garner, W. R., \& Clement, D. E. Goodness of pattern and pattern uncertainty. Journal of Verbal Learning and Verbal Behavior, 1963, 2, 446-452.

Goldmeier, E. [Similarity in visually perceived forms] (E. Goldmeier, trans.). Psychological Issues, 1972, 8(Whole No. 29), 1-135. (Originally published, 1937.)

GotTschaldT, K. Über den Einfluss der Erfahrung auf die Wahrnehmung von Figuren, I. Psychologische Forschung, 1926, 8, 261-317.

Hock, H. S. The effects of stimulus structure and familiarity on same-different comparison. Perception \& Psychophysics, 1973, 14, 413-420.

Kroll, J. F., \& Hershenson, M. Two stages in visual matching. Canadian Journal of Psychology, 1980, 34, 49-61.

МАCH, E. Contributions to the analysis of sensations. Chicago: Open Court, 1897.

Rock, I. Orientation and form. New York: Academic Press, 1973.

RYDER, J. Symmetry relations in visual matching. Unpublished doctoral dissertation, Brandeis University, 1979.

Sekuler, R. W., \& Houlihan, K. Discrimination of mirror images: Choice time analysis of human adult performance. Quarterly Journal of Experimental Psychology, 1968, 6, 225-233.

Vitz, P. C., \& Todd, T. C. A model of the perception of simple geometric figures. Psychological Review, 1971, 78, 207-228.

WEYL, H. Symmetry. Princeton, N.J: Princeton University Press, 1952.

WhITE, M. J. Naming and categorization of tilted alphanumeric characters do not require mental rotation. Bulletin of the Psychonomic Society, 1980, 15, 153-156.

WolfF, P. Mirror-image confusability in adults. Journal of Experimental Psychology, 1971, 91, 268-272.

Zusne, L. Measures of symmetry. Perception \& Psychophysics, 1971, 9, 363-366.

\section{NOTE}

1. A number of matching experiments have been reported using pairs of symmetrical stimulus elements (e.g., Corballis \& Roldan, 1974; Egeth, Brownell, \& Geoffrion, 1976; Sekuler \& Houlihan, 1968; Wolff, 1971). When these stimuli are categorized in terms of the definitions above, however, it is clear that the comparisons were only among stimuli showing different types of symmetry. The stimuli used in the present experiments were different because, first, the elements were not symmetrical and, second, the total stimulus set included both symmetrical and asymmetrical stimuli (considering all forms of symmetry).

(Received for publication October 16, 1981.) 